\title{
Simulation model of situational control of transshipment machinery fleet
}

\author{
Igor Zub ${ }^{1, *}$, Yuriy Ezhov ${ }^{1}$, and Viktor Shchemelev ${ }^{1}$ \\ ${ }^{1}$ Admiral Makarov State University of Maritime and Inland Shipping, 5/7, Dvinskaya str, 198035, \\ Saint-Petersburg, Russia
}

\begin{abstract}
The seaport is a place for cargo transshipment and storage. The speed of vehicle processing is one of the indicators of port's performance. The time of vehicles being under processing depends on the performance of a fleet of transshipment machinery. The stochastic nature of vehicles' arriving leads to the intense operation of handling facilities and formation of wait lists for vehicles to be loaded or unloaded, or to the downtime of transshipment machinery when vehicles are absent. Depending on the operational situation, transshipment machinery is brought out for maintenance operations that can be performed by unit, which provides the increase in the technical use coefficient. For maintenance of individual units, the decomposition of handling facility is performed; statistical data on the time of maintenance conduction for all units is collected. Statistical data are entered into the database. When transshipment machinery is brought out of operation for a short period, the unit is selected, the service time of which coincides with the downtime of a brought out facility. To visualize the process of situational management of maintenance and define conflict situations, a simulation model was developed with the use of the apparatus of Petri nets. The analysis of the model helped to reveal conflict situations that can be solved both during situational management of transshipment machinery fleet and by means of models nested into conflict places.
\end{abstract}

\section{Introduction}

Seaport is a place for transshipment, storage and dispatch of cargo to a recipient. Arriving and departure of cargo is performed by various means of transport (water, railroad, motor and pipeline transport). Interaction of different modes of transport entails the involvement of both deterministic and stochastic components for their processing.

The aim of a seaport is to perform loading and unloading works $(L U W)$. The follow-up rate of a transportation facility $(T F)$ is one of quality indicators of a seaport. Both $L U W$ and processing of $T F \mathrm{~s}$ depend on technical condition of transshipment machinery (TM), therefore the issue of organization and control of maintenance works is rather relevant. The time of $T F$ processing $\left(T_{T F}\right)$ is a function depending on several variables [1]:

$$
T_{L U D}=f\left(F_{T M}, P_{T M}, Q_{p}, A T M\right),
$$

\footnotetext{
* Corresponding author: zubiv@mail.ru
} 
where $F_{T M}$ - fleet of transshipment machinery; $P_{T M}$ - performance of transshipment machinery; $Q_{p}$ - qualifications of operating personnel; $A T M$ - administrative and technical measures.

Administrative and technical measures enable maintaining $T M$ in operable condition with minimal economic costs [2]. These measures include the preparation of maintenance works. It should be noted here that maintenance in accordance with GOST 18300-2016 "System of maintenance and repair. Terms and definition" can be divided into several stages, the operating time demanded for conducting maintenance can be increased beyond the established by the manufacturer, repairs can be carried out both according to schedule and proceeding from technical condition. Some authors $[3,4]$ propose to transit from procedural maintenance works to those based on the actual state of vehicles, but this position is not completely correct, since maintenance is performed in order to keep operational performance. When transiting to maintenance strategy based on the actual state of vehicles, the number of emergency repairs will increase. In work [5], it is proposed to carry out routine maintenance proceeding from the actual state of operational materials, which significantly reduces financial costs and downtime during maintenance work. The existence of different views on the solution of the maintenance problem shows the necessity of a search for a new optimal strategy for conducting routine maintenance.

When operating technical systems, it is known that along the observed trajectory $X_{k}$, at time $t_{k}$, only two decisions can be made [6]:

- not to interfere with the system performance and continue to monitor the process of changing the parameter $X(t)$;

- to stop the operation of a system and return the system to its original state through routine maintenance.

The present paper considers the management of maintenance work depending on the current production situation of a port. In most cases, the arrival of a transport mode for processing is stochastic, and is determined by a certain time "window". There is an opportunity to perform maintenance of a separate mechanism within the occurred "window". In this case, $T M$ is not decommissioned for routine maintenance, the processing quality of a vehicle does not decrease, while the coefficient of technical operation $\left(C_{T O}\right)$ increases.

To conduct maintenance within the "windows", it is necessary to decompose $T M$ into separate mechanisms [7]. Based on the available statistical data, the average time of maintenance for each mechanism is determined, which allows planning works for the existing period or minimizing downtime for a vehicle arrived for processing. When a window occurs, the maintenance service informs the repair service of the time interval they have. The repair service plans scheduled maintenance in accordance with the available statistics.

The implementation of the strategy for conducting maintenance during time "windows" is possible with permanent monitoring, which gives complete information on the technical condition of a $T M$, since each $T M$ unit has uncertainty. The degree of uncertainty in this case is not significant, it is important to know only the probability of a transition from one technical condition to another [8]. Permanent monitoring is carried out using bot computers that process information coming from sensors installed on the units. Modern information technologies allow the remote monitoring of the main units. Here, operating conditions of $T M$ fleet should be taken into account that can entail the fail of not only units, but also diagnostic equipment. Parts and units, from which it is not possible to take data remotely, are inspected visually during breaks and routine maintenance. This places high demands on technical service workers, and is one of the reasons for transferring maintenance of equipment to outsourcing. 


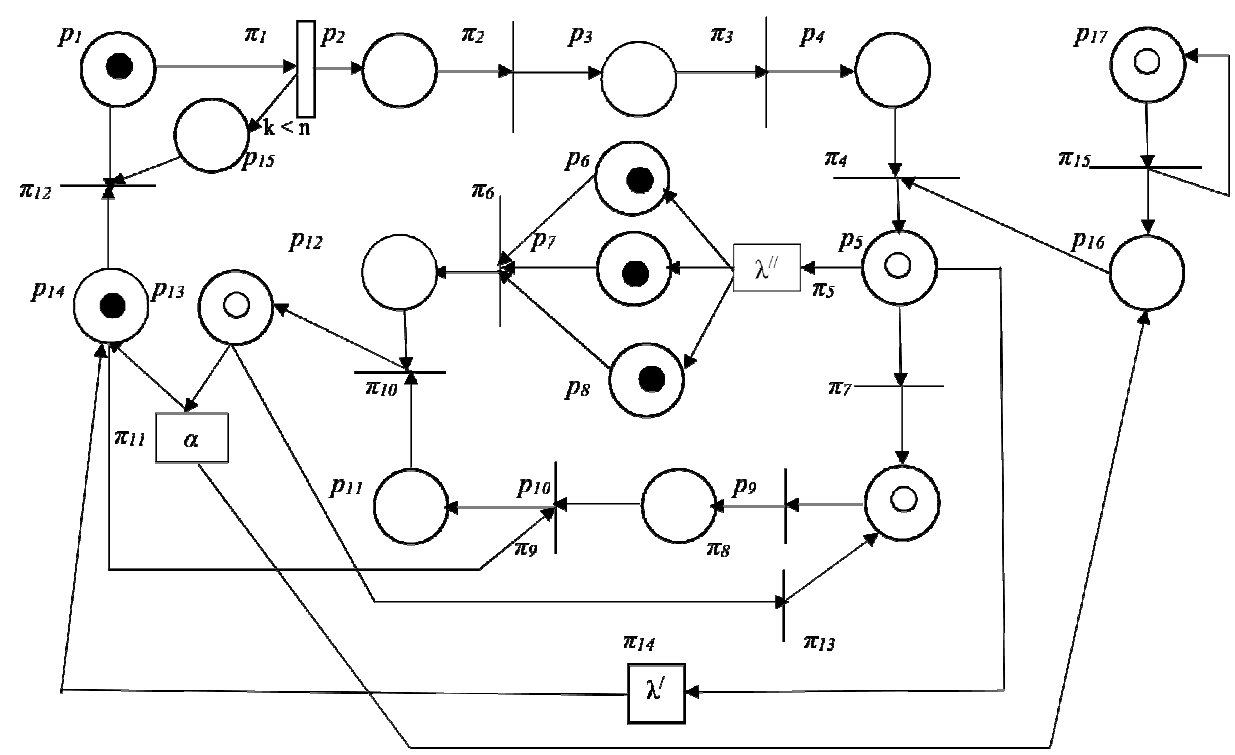

Fig. 1. Simulation model of bringing out a $T M$ to maintenance $p_{1}-T F ; p_{2}-$ information on "window" occurring; $p_{3}$ - time of a "window"; $p_{4}$ - information is received; $p_{5}-T M$ is defined; $p_{6}$ - human resources; $p_{7}$ - replacement parts; $p_{8}$ - combustibles and lubricants; $p_{9}$ - database of time for maintenance for individual mechanisms; $p_{10}-$ mechanism is defined; $p_{11}-T M$ is brought out to maintenance; $p_{12}$ - resources are defined; $p_{13}$ - maintenance is performed; $p_{14}-T M$ fleet; $p_{15}$ - place of processing; $p_{16}$ - time mechanism; $p_{17}$ - "clockwork pendulum"; $\pi_{1}$ - arriving of a $T F$ to the port; $\pi_{2}$ - determination of time of a «window»; $\pi_{3}$ - information transfer to the repair service; $\pi_{4}-$ defining a $T M$ with operation time for maintenance; $\pi_{5}-$ preparation for maintenance; $\pi_{6}-$ determination of the sufficiency of resources; $\pi_{7}$ - search in the database of the mechanism for which the time for maintenance corresponds to the time of "window"; $\pi_{8}-$ mechanism selection; $\pi_{9}-$ bringing out of $T M$ to maintenance; $\pi_{10}$ - performance of maintenance; $\pi_{11}$ - putting $T M$ into operation; $\pi_{12}$ - processing of $T F ; \pi_{13}$ - entering information into the database; $\pi_{14}-$ transfer of information to the operator about $T M$ 's bringing out; $\pi_{15}$ - pendulum operation.

\section{Research methods}

A number of specific features of the maintenance management of $T M$ fleet of a port determines the use of simulation modelling when optimizing information management technologies:

- traffic flows and the process of servicing applications are stochastic;

- structure of the organization of routine maintenance distinguishes subsystems with feedbacks;

- optimization of individual subsystems can lead to conflict situations and does not provide system optimization;

- system optimization of technical service of a port cannot be implemented under one mathematical programming task.

To visualize the management of maintenance operations, a simulation model based on Petri nets was developed (Fig. 1). Modeling management processes can improve the quality of work, identify problems that may arise and find ways to address them. The use of the apparatus of Petri nets has the following prerequisites: the presence of deterministic and stochastic components in TM's arrival for processing; the ability to perform maintenance works in the form of sets of parallel processes; situational management associated with the stochasticity of the input flow of $T M \mathrm{~s}$ and the current operating time of $T M$ fleet. 
The Petri net is a directed graph, the vertexes of which (places and transitions) are interconnected by arcs. Tokens that control the transitions and are moved around the network are located in places. The transition is triggered by removing a token from its input place and the formation of new tokens located in its input places. To simulate the process of managing maintenance operations over $T M$, extensions of Petri nets are used, such as nested Petri nets, which represent the structure and behavior of complex systems, since the tokens in the system network are Petri nets themselves and have their own behavior $[9,10]$.

\section{$3 \quad$ Results}

The simulation model of preparing $T M$ for maintenance during time "windows" is not simple, since there are places in the network where conflict situations arise. Conflict situation imply such a situation in the Petri net when the place has outputs for two or more transitions. Depending on the choice, only one transition can be activated, because starting a transition will remove a token from its place so other transitions cannot be started.

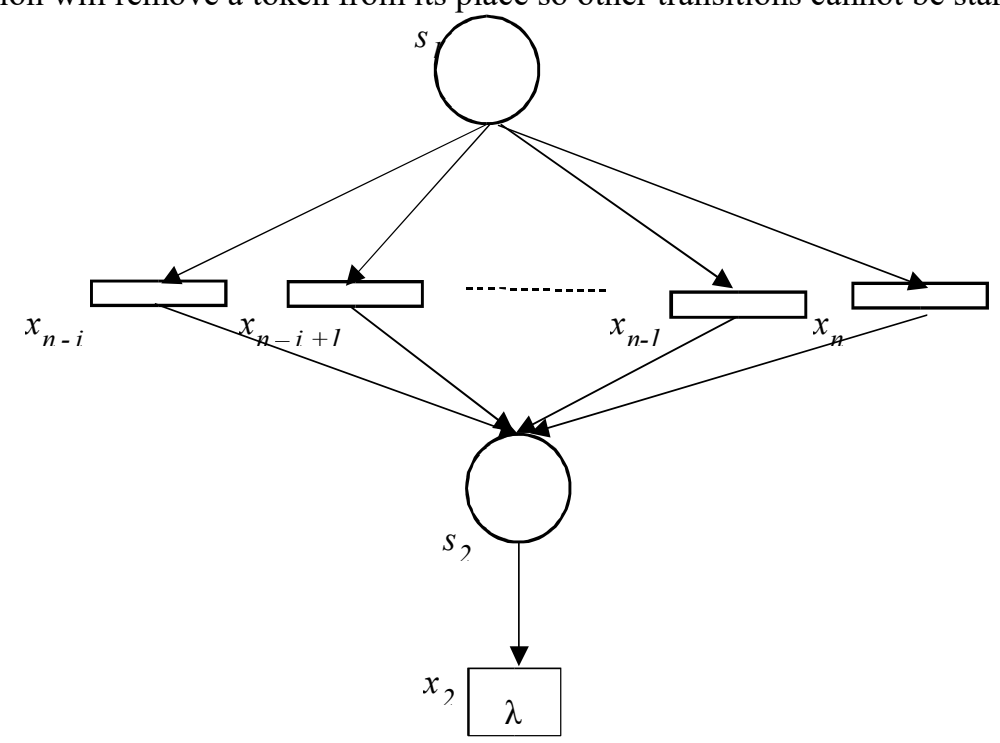

Fig. 2. Embedded network of place $p_{5}, s_{1}$ - working hours counters of $T M ; s_{2}$ - correspondence of operation time for maintenance; $x_{n-i}, x_{n-i+1}, \ldots, x_{n-1}, x_{n}$ - reading hour meter of $T M ; x_{1}$ - maintenance number determination

In place $p_{l}$, a token has dimension $k$, which reflects the average number of $T F$ s arriving at the port during the day. Place $p_{l}$ is a conflicting one. The Petri net shows potential choices here [11]. The conflict is resolved through situational management by a decision maker. The following are aspects are related to situational management:

- determination of the current situation and its comparison with the shift-daily plan of the port;

- assessment of the actual situation;

- probabilistic outcomes of the situation;

- preventive measures in accordance with the current situation to prevent negative consequences;

- adoption of a management decision.

Depending on the situation, a token from place $p_{l}$ can trigger the transition of both $\pi_{l}$ and $\pi_{12}$. Transition $\pi_{1}$ is a transition with protection (according to the terminology [9]). 
Protection possesses a value: $k<n$, where $k$ is the number of vehicles arriving at the port, $n$ is the quantitative composition of $T M$ fleet. With a decrease in the $T F$ flow to the port, a forced downtime of $T M \mathrm{~s}$ occurs. In place $p_{5}$ there is a conflict situation. Place $p_{5}$ has an integrated network (Fig. 2). Transition $x_{2}$ (Fig. 2) has marker $\lambda$. Transition $\pi_{14}$ has marker $\lambda^{\prime}$, transition $\pi_{5}$ has marker $\lambda^{\prime \prime}$, which means that these markers complement each other and all marked transitions can work simultaneously [9].

When determining the downtime of a "window", a TM unit is brought out for maintenance. Maintenance conduction demands tokens located in places $p_{6}, p_{7}, p_{8}$, that indicate the resources necessary for maintenance, otherwise, when tokens are absent, $T M$ will not be brought out for routine maintenance. In the second case, the input stream of $T F \mathrm{~s}$ will be serviced until the inequality $k<n$ is reached. This will happen when the input stream of $T F \mathrm{~s}$ is not replenished until it is completely exhausted.

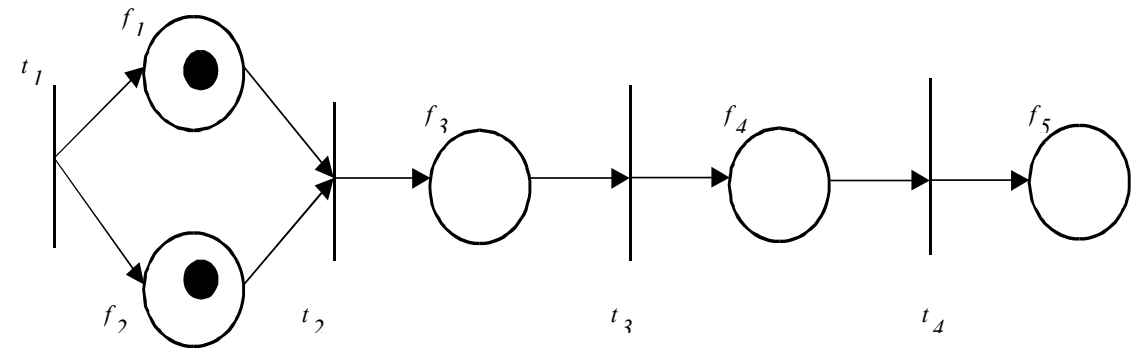

Fig. 3. Embedded network of place $p_{9}, f_{1}$ - repair personnel; $f_{2}$ - mechanism of $T M ; f_{3}$ - maintenance is performed; $f_{4}$ - information about the time of maintenance in person-hours; $f_{4}$ - database; $t_{1}-$ maintenance planning; $t_{2}$ - performing of maintenance; $t_{3}$ - recording the time of maintenance in person-hours; $t_{3}$ - entering information into the database.

Place $p_{9}$ has an integrated Petri net (Fig. 3), simulating the formation of a database by the time of maintenance of individual mechanisms. In place $p_{14}$, a token has dimension $n$, showing the quantitative composition of the $T M$ fleet and, therefore, introduces a network restriction.

In places $f_{1}$ and $f_{2}$ (Fig. 3) there are tokens that show the availability of resources necessary for the performance of work. At the end of the work, the time for completing maintenance is recorded and recalculated in man-hours. Then this information is entered into the database. The database allows selecting a $T M$ mechanism, which has enough operating time to be maintained, in order to conduct routine maintenance in a certain period.

Place $p_{13}$ is a conflict one, the conflict in this place is resolved by the embedded network (Fig. 4).

Maintenance with decomposition of $T M$ into separate mechanisms and the collected information database can increase both the coefficient of technical use and the coefficient of technical readiness of $T M$. As a result of the organizational and technical measures, downtimes of $T M$ for carrying out scheduled activities are reduced, the need for the reserve of $T M \mathrm{~s}$ is reduced, and the quality of processing of vehicles is improved. 


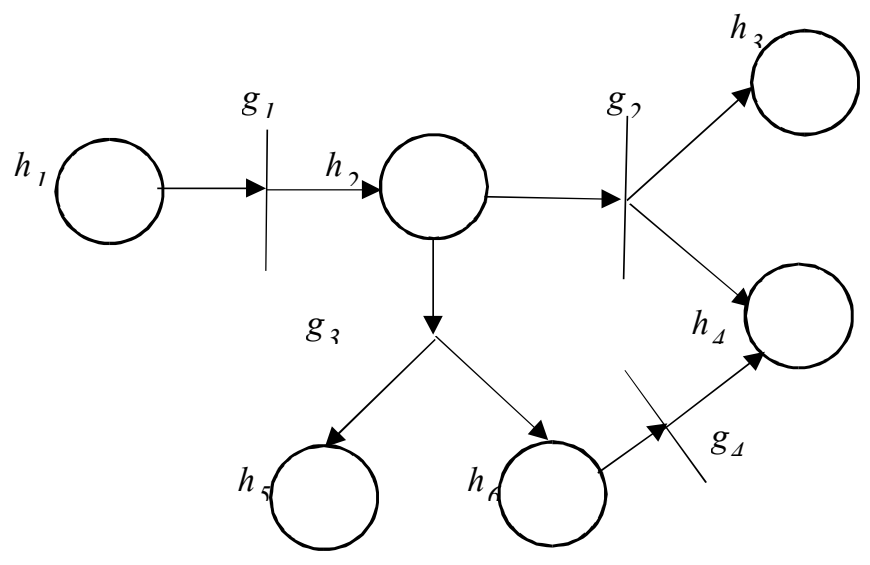

Fig. 4. Embedded network of place $p_{13}, h_{1}$ - maintenance works completed; $h_{2}$ - diagnostics completed; $h_{3}$-operation service; $h_{4}$ - database; $h_{5}$ - the decision to bringing out a TM for repair; $h_{6}-$ defect report; $g_{1}$ - diagnostics of $T M ; g_{2}$ - transmission of information on completion of maintenance; $g_{3}$ - diagnostic data analysis; $g_{4}$ - repair planning

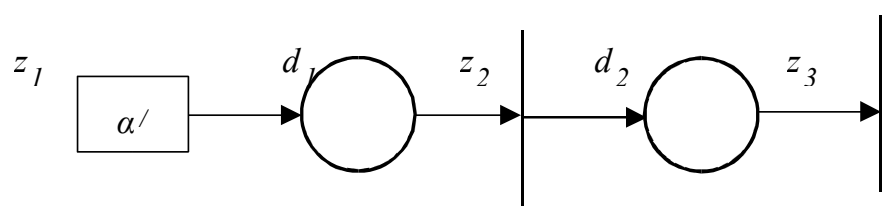

Fig. 5. Embedded network of place $p_{15}, d_{1}$ - "clockwork pendulum" $d_{2}-$ operating time of $T M ; z_{1}-$ turning on of "clockwork pendulum"; $z_{2}$ - fixation of operating time of $T M ; z_{3}$ - operating time data transfer

\section{Discussion}

The initial marking of the network contains tokens $p_{1}, p_{5}, p_{6}, p_{7}, p_{8}, p_{9}, p_{13}, p_{14}, p_{17}$. In places $p_{5}, p_{9}, p_{13}, p_{17}$, tokens have their nested networks. Places $p_{6}, p_{7}, p_{8}, p_{14}$ characterize the resources of the port.

Considering the network model of organizing the maintenance of $T M$ fleet as a hierarchy of nested networks, the assumption can be made that at the top level of the hierarchy, all or several tokens designate a descending cascade of private networks that describe the implementation of certain functions. The important feature of the proposed modeling approach is the description of each token by its own "nested" (according to terminology [9]) network. Nested networks allow a compositional analysis of the model [12].

Petri nets, being an asynchronous modeling tool, reflect only cause-effect relations in the object and the logic of model's functioning. For concretization, the asynchronous Petri net is combined with time characteristics of maintenance work operations. For this purpose, each transition $\pi_{j}$, which designates an individual production operation, is assigned with the time of its implementation $\tau_{j}$ in the simulation algorithm. The value of $\tau_{j}$ can be set within the simulation in two ways: either as the average sample in the aggregate of statistical data, or by playing out the corresponding empirical distribution function. 
The second approach implies that a real-time counter is integrated into the network as a set of network fragments related to individual operations. This implies a clock mechanism in the network ("pendulum") which makes values of all durations integral [13]. In the model (Fig. 1), a network is introduced consisting of places $p_{15}, p_{16}$ and transition $\pi_{15}$, the task of which is to record the response time of transition $\pi_{14}$ - putting TM into operation and starting transition $\pi_{4}$. Place $p_{15}$ has an integrated network (Fig. 5). The network of place $p_{15}$ monitors the operating time of TM after its commissioning. On the one hand, it is the duplication of the meter of running hours, on the other hand, it is the automatic recording of operating time, because the meter is read off by an operator, so there is a possibility of error in exporting data on paper. Transition $z_{l}$ has marker $\alpha$. Marker $\alpha$ is in transition $\pi_{1 l}$. The start of transition $\pi_{11}$ triggers transition $z_{1}$, which leads to starting a countdown of the operating time.

The introduction of a temporary network into the model allows controlling the time characteristics, which enables the automatic tracking of both operating time for maintenance and the time spent on maintenance of individual $T M$ mechanisms. The obtained statistics allow improving the quality of routine maintenance planning and the planning of workload of repair personnel.

Analyzing the Petri net (Fig. 1), the following conclusions can be drawn: the network is not safe, because in places $p_{1}$ and $p_{14}$, tokens have $>1$ dimension, which indicates that the network can be launched before the completion of the previous launch. When the port's capabilities for processing $T F \mathrm{~s}$ is known, the quantity of vehicles that can arrive at the port for processing is determined, then in place $p_{l}$ a limit for token number $k$ is set. The network becomes $k$-secure or limited. If the flow of vehicles to the port increases, only $k$ units of $T F \mathrm{~s}$ will be processed. With a decrease in the flow of vehicles, the model is turned on to bring the next $T M$ unit to maintenance, even if $T M$ is currently under maintenance. This event will occur only if there are a sufficient amount of resources in places $p_{6}, p_{7}, p_{8}$. In this case, maintenance workflows will be parallelized [14].

Security of the model is ensured by introducing a restriction in place $p_{l}$, this place cannot contain more than $k$ tokens. When this condition is not met, vehicles will stand idle in line for processing.

The Petri net (Fig. 1) is non-preserving, since in places $p_{6}, p_{7}, p_{8}, p_{14}$ the number of tokens can change: human resources (vacations, illnesses, other reasons); replacement parts, fuels and lubricants (not timely replenishment of a storehouse); change in the quantitative composition of $T M$ fleet.

Since all transitions can be triggered by any layout, therefore the network is alive.

There are several methods for the analysis of Petri nets, but the matrix method is the most promising for the analysis of real systems [15]. To analyze the attainability of marking, the structure of the Petri net is represented in the form of two incident matrices $D^{+}$ and $D^{-}$, with the number of rows equal to the number of transitions in the network and the number of columns equal to the number of places. $D^{-}$is the input matrix containing 1 at the intersection of the $j$-th row and the $i$-th column, when the $i$-th place is the input for the $j$-th transition, or 0 otherwise. $D^{+}$is the matrix of outputs containing 1 at the intersection of the $i$-th row and the $j$-th column, when the place is the output for the $i$-th transition. 


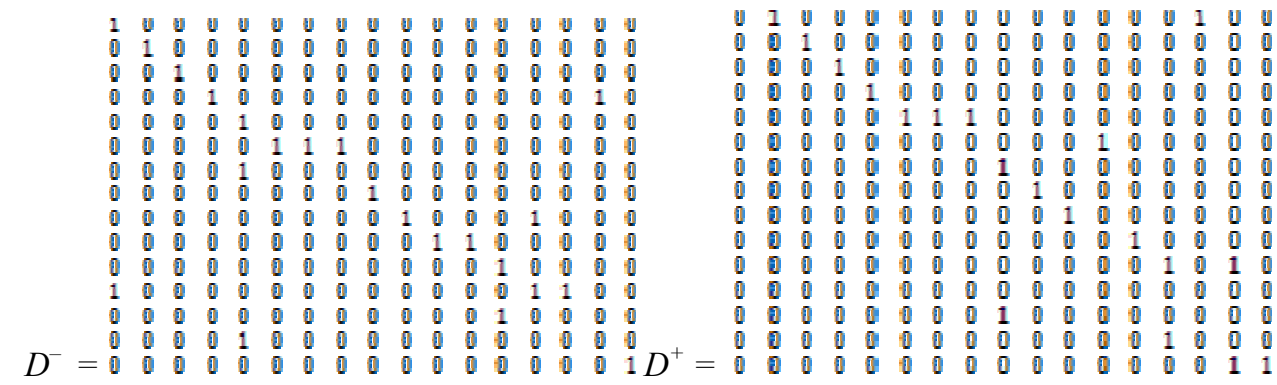

To analyze the net, the change matrix is to be found:

$$
D=D^{+}-D^{-} \text {. }
$$

$\begin{array}{ccccccccccccccccc}-1 & 0 & 0 & 0 & 0 & 0 & 0 & 0 & 0 & 0 & 0 & 0 & 0 & 0 & 1 & 0 & 0 \\ 0 & -1 & 1 & 0 & 0 & 0 & 0 & 0 & 0 & 0 & 0 & 0 & 0 & 0 & 0 & 0 & 0 \\ 0 & 0 & 1 & 1 & 0 & 0 & 0 & 0 & 0 & 0 & 0 & 0 & 0 & 0 & 0 & 0 & 0 \\ 0 & 0 & 0 & -1 & 1 & 0 & 0 & 0 & 0 & 0 & 0 & 0 & 0 & 0 & 0 & -1 & 0 \\ 0 & 0 & 0 & 0 & -1 & 1 & 1 & 1 & 0 & 0 & 0 & 0 & 0 & 0 & 0 & 0 & 0 \\ 0 & 0 & 0 & 0 & 0 & -1 & -1 & -1 & 0 & 0 & 0 & 1 & 0 & 0 & 0 & 0 & 0 \\ 0 & 0 & 0 & 0 & -1 & 0 & 0 & 0 & 1 & 0 & 0 & 0 & 0 & 0 & 0 & 0 & 0 \\ 0 & 0 & 0 & 0 & 0 & 0 & 0 & 0 & -1 & 1 & 0 & 0 & 0 & 0 & 0 & 0 & 0 \\ 0 & 0 & 0 & 0 & 0 & 0 & 0 & 0 & 0 & -1 & 1 & 0 & 0 & 0 & 0 & 0 & 0 \\ 0 & 0 & 0 & 0 & 0 & 0 & 0 & 0 & 0 & 0 & -1 & -1 & 1 & 0 & 0 & 0 & 0 \\ 0 & 0 & 0 & 0 & 0 & 0 & 0 & 0 & 0 & 0 & 0 & 0 & -1 & 1 & 0 & 1 & 0 \\ -1 & 0 & 0 & 0 & 0 & 0 & 0 & 0 & 0 & 0 & 0 & 0 & 0 & -1 & -1 & 0 & 0 \\ 0 & 0 & 0 & 0 & 0 & 0 & 0 & 0 & 1 & 0 & 0 & 0 & 1 & 0 & 0 & 0 & 0 \\ 0 & 0 & 0 & 0 & -1 & 0 & 0 & 0 & 0 & 0 & 0 & 0 & 0 & 1 & 0 & 0 & 0 \\ 0 & 0 & 0 & 0 & 0 & 0 & 0 & 0 & 0 & 0 & 0 & 0 & 0 & 0 & 0 & 1 & 0\end{array}$

Transition $\pi_{i}$ in marking $\varphi$ is allowed when $\varphi \geq e[\mathrm{i}] D^{-}$and the result of start is written as follows:

where $e[i]$ is a row-vector.

$$
\delta\left(\varphi, \pi_{i}\right)=\varphi+e[i] D
$$

To determine the persistence of the Petri net, non-zero weighting vector $\omega$ is to be found: the column vector for which the weighted sum over all attainable markings is constant. The necessary condition $\varphi \omega=\varphi^{\prime} \omega$. Since $\varphi^{\prime}$ is attainable, a sequence of triggers of transitions $\sigma$ exists that takes the network from $\varphi$ to $\varphi^{\prime}$. Therefore, $f(\sigma) D \omega=0$. Since it is true for all $f(\sigma)$, the following equation proceeds:

$$
D \omega=0 \text {. }
$$

Petri net is persisting if a positive vector $\omega$ exists, for which equality (4) is true, which provides an algorithm for checking persistence and allows one to obtain the weighting vector $\omega[16]$.

Using the matrix theory of Petri nets, the problem of attainability is solved. There is a sequence of triggers of transitions $\sigma$, which leads to $\varphi^{\prime}$ from marking $\varphi$. This means that $f(\sigma)$ is a non-negative integer solution of the following matrix equation for $x$ :

$$
\varphi^{\prime}=\varphi+x D \text {. }
$$

If $\varphi^{\prime}$ can be attained from $\varphi$, then equation (5) has a solution in non-negative numbers, if equation (5) has no solution, then $\varphi^{\prime}$ is unattainable from $\varphi$.

Petri nets reflect the logic of technological processes and analysis of both organizational and technical systems, which implies that the use of Petri nets in the development of technological and organizational processes allows not only to visualize these processes, but also to optimize them at the stage of their development. Modeling of technological processes of maintenance, as well as obtaining operational information on technical condition of $T M$ received from permanent diagnostics, allows optimizing routine maintenance and implementing strategies for maintenance within time "windows". Such a 
strategy increases the coefficient of technical utilization and reduces the number of reserve machinery, which consequently reduces the capital investments of the port.

\section{References}

1. I. Zub, YU. Yezhov, Logistics: current development trends: materials of the KhVI International Scientific and Practical Conference. 161-164. (2017)

2. I. Zub, YU. Yezhov, Logistics: current development trends: materials of the KhVI International Scientific and Practical Conference. 190-193. (2018)

3. A.A. Lisov, S.A. Izilov, Basic research. N 8-2. 386-388. (2011)

4. S.P. Ozorin, I.Ye. Berdnikov Vestnik Zabaykal'skogo gosudarstvennogo universiteta. N 8 (111). P. 64-69. (2014)

5. A.L. Manakov, A.YU. Kirpichnikov, T.K. Tyunyukova Vestnik IrGTU. N 5. 127-131. (2015)

6. D.V. Varnakov, O.N. Didmanidze, Agrarnaya nauka Yevro-Severo-Vostoka. N 2 (57). 67-71. (2017)

7. S.S. Sokolov, YU.Ye. Yezhov, I.V. Zub Rechnoy transport (XXI vek). N 4, 52-58. (2016)

8. V.D. Vereskun, A.L. Manakov, A.YU. Kirpichnikov. Mir transporta. N 3. 4-9. (2012)

9. I.A. Lomazova Vlozhennyye seti Petri: modelirovaniye i analiz raspredelonnykh sistem s ob"yektivnoy strukturoy. 208 p. (2004)

10. V.O. Yermakova, I.A. Lomazova Trudy ISP RAN. vol 28. issue 4. 115-136. (2016)

11. S.A. Yuditskiy, Avtomatika i telemekhanika. N 1. 114-123. (2008)

12. R.A. Nesterov, I.A. Lomazova Trudy ISP RAN. vol. 29, issue 4, 21-38. (2017)

13. A.A. Leskin, P.A. Mal'tsev, A.M. Spiridonov, Seti Petri v modelirovanii $i$ upravlenii. (1989)

14. V. van der Aalast, K. van Khey. Workflow Management: Models and Systems. Moscow, Fizmatlit (2007)

15. D.A. Zaytsev Cybernetics and Systems Analysi. N 1. 143-154. (2006)

16. J. Piterson, Theory of Petri Nets and System Modeling, Moscow, Mir (1984) 